OPEN ACCESS

Edited by: Mark Hallahan,

College of the Holy Cross, USA

Reviewed by:

Liudmila Liutsko,

Barcelona Biomedical Research Park,

Spain

Marcus Rothkirch,

Charité - Universitätsmedizin Berlin,

Germany

*Correspondence:

Hong Chen

chenhg@swu.edu.cn;

Hui Kou

kouhuiswu@126.com

tThese authors have contributed equally to this work.

Specialty section:

This article was submitted to Personality and Social Psychology, a section of the journal

Frontiers in Psychology

Received: 15 March 2016 Accepted: 03 June 2016

Published: 27 June 2016

Citation:

Kou H, Su Y, Bi T, Gao X and Chen $H$ (2016) Attentional Biases toward Face-Related Stimuli among Face Dissatisfied Women: Orienting and Maintenance of Attention Revealed by Eye-Movement. Front. Psychol. 7:919. doi: 10.3389/fpsyg.2016.00919

\section{Attentional Biases toward Face-Related Stimuli among Face Dissatisfied Women: Orienting and Maintenance of Attention Revealed by Eye-Movement}

\author{
Hui Kou ${ }^{1 *}$, Yanhua Su${ }^{1,2 t}$, Taiyong Bi ${ }^{1}$, Xiao $\mathrm{Gao}^{1}$ and Hong Chen ${ }^{\text {* }}$ \\ ' Faculty of Psychology, Southwest University, Chongqing, China, ${ }^{2}$ School of Management, Zunyi Medical University, Zunyi, \\ China
}

The present study was aimed to examine attentional biases toward attractive and unattractive faces among face dissatisfied women. Twenty-seven women with high face dissatisfaction (HFD) and 27 women with low face dissatisfaction (LFD) completed a visual dot-probe task while their eye-movements were tracking. Under the condition of faces-neutral stimuli (vases) pairs, compared to LFD women, HFD women directed their first fixations more often toward faces, directed their first fixations toward unattractive faces more quickly, and had longer first fixation duration on such faces. All participants had longer overall gaze duration on attractive faces than on unattractive ones. Our behavioral data revealed that HFD women had difficulty in disengaging their attention from faces. However, there are no group differences in stimulus pairs containing an attractive and an unattractive face. In sum, when faces were paired with neutral stimuli (vases) HFD women showed an attention pattern characterized by orienting and maintenance, at least initially, toward unattractive faces but showed overall attention maintenance to attractive ones, but any attention bias wasn't found in attractive unattractive face pairs.

Keywords: dot probe paradigm, attention bias, facial attractiveness, facial dissatisfaction, eye-movement

\section{INTRODUCTION}

Facial attractiveness plays a central role in social activities, such as job hunting (Desrumaux et al., 2009), mate choice (McNulty et al., 2008), and interpersonal communication (Thornhill and Gangestad, 1999). Face dissatisfied individuals refer to those who show high levels of facial appearance concerns, produce negative cognition, and express negative emotion about their facial appearance, and therefore they will adopt relevant adjustments because of their unattractive faces (Rumsey and Harcourt, 2005). In this study, we used the face subscale of The Negative Physical Self Scale (Chen et al., 2006b) to identify women with high face dissatisfaction (HFD) and women with low face dissatisfaction (LFD) ${ }^{1}$.

\footnotetext{
${ }^{1}$ High face dissatisfaction refers to high face dissatisfaction, and LFD refers to low face dissatisfaction.
} 
Previous studies almost exclusively focused on body image dissatisfaction and eating disturbance, which revealed visual attention biases toward body-related stimulus based on sample differences in reaction time (RT), whether in Western culture (Ben-Tovim and Walker, 1991; Lee and Shafran, 2004) or in Eastern culture (Liang et al., 2008; Feng et al., 2010; Gao et al., 2011a,b, 2012). However, it was worth noting that Chinese adolescents and young adults expressed relatively more concerns about face appearance than about fatness (Chen et al., 2006b). What's more, facial dissatisfaction could be regarded as an important part of body image dissatisfaction. Thus, it is of great importance to study spatial attention bias toward face-related stimulus with HFD based on the abovementioned research evidence.

Nevertheless, attention is a complicated cognitive process, including orientation, maintenance, disengagement and shift (Posner and Petersen, 1990). Moreover, each component of attention could dominate at a certain stage of attention Previous behavioral experiments using attention paradigms could only investigate one or two components of attention, such as attention orientation in the Stroop task (Ben-Tovim and Walker, 1991) and attention orienting and attention maintenance in the spatial cueing paradigm (Feng et al., 2010). The above paradigms could not reveal dynamical course of attention.

Researchers found that the eye-movement tracking technique provided a sensitive indicator for initial orienting, initial shift and maintenance of attention (Caseras et al., 2007). Some studies found that when viewing their own body, the eating disorders group focused less on their own self-defined "beautiful" body parts and more on their "ugly" parts than the control group did but showed the opposite pattern when viewing other people's bodies (Jansen et al., 2005). Another study indicated that weight dissatisfied women directed their initial gaze toward fatness words, had a shorter mean latency of first fixation on both fatness and thinness words, had longer first fixation on fatness words but shorter first fixation on thinness words, and had shorter total gaze duration on thinness words (Gao et al., 2011b). Gao et al. (2012) further employed the technique of eye-movement in a dot probe experiment to explore attentional bias toward body-related pictures among females with weight dissatisfaction, and they found that an orienting-maintenance pattern of attention toward fatness-related pictures emerged among these women. It implied that individuals with body image dissatisfaction preferentially attended to body-related or fatnessrelated stimuli.

However, so far, attentional bias toward face-related stimulus among HFD females has not yet been studied, although facial dissatisfaction was also an important sub-component of body image dissatisfaction. Clinical research has revealed an attentional bias toward face-related stimuli among patients with body dysmorphic disorder (BDD) characterized by a distressing or impairing preoccupation with an imagined or slight defect in appearance that causes clinically significant distress or functional impairment (Didie et al., 2010). A study using the Stroop task indicated that, compared to controls, BDD patients were more distracted by face-related words (e.g., disfigured), with the greatest interference from positive face-related words (Buhlmann et al., 2002). However, another eye-movement study revealed that $\mathrm{BDD}$ patients showed increased selective attention to perceived defects in their own faces and unfamiliar faces (Grocholewski et al., 2012). These contradictory results might derive from inconformity in paradigms and stimulus. Using a dot probe task, researchers found that dysmorphic concern was positively correlated with attention to faces and attractive facerelated images during the long stimulus presentation $(1000 \mathrm{~ms})$, whereas during the short stimulus presentation (200 ms), dysmorphic concern was positively correlated with disgusting images (Onden-Lim et al., 2012). We could conclude from this study that different attention components might play different roles at specific stages of attention course. More interestingly, considering that body image dissatisfaction has been considered to be a core feature of BDD (Cororve and Gleaves, 2001), studies of attentional bias toward faces among BDD patients could lay a solid foundation for attentional bias studies among HFD samples.

It is of great importance to study attentional biases toward faces among HFD individuals for two reasons. First, we may be able to further apply the model of body image dissatisfaction to faces. Secondly, we may be able to accordingly develop corresponding interventions to reduce distress for these people. Given that young females are more concerned with facial attractiveness than males in China (Chen et al., 2006a), we only recruited young females as our experimental participants. In the present study, we aimed to investigate the difference of spatial attentional biases between HFD women and LFD women using the eye movement-tracking technique. According to previous studies (Gao et al., 2011b, 2012), we hypothesized that in comparison with the LFD group, HFD women would be more likely to locate their initial fixation on unattractive faces, direct their first gaze faster to unattractive faces, have longer first fixation on unattractive faces, but have shorter first fixation on attractive faces, and have longer total gaze duration on unattractive ones. Regarding behavioral data, we predicted that HFD women might have difficulty in attentional disengagement from unattractive faces.

\section{MATERIALS AND METHODS}

\section{Participants}

Our research sample was composed of 27 HFD women (age, $M=20.19, S D=1.86)$ and 27 LFD female undergraduate students (age, $M=20.52, S D=1.72$ ) with ages ranging from 18 to 24 years old. The Negative Physical Self Scale-Face subscale (NPSS-Face) was employed to discriminate whether a participant had HFD or LFD (Gao et al., 2012). Only those who scored higher than 27.5 (HFD, $M=29.33, S D=2.91$ ) were considered to be HFD women; those who scored lower than 16.5 (LFD, $M=6.93$, $S D=2.13$ ) were regarded as LFD women. According to selfreport, all of the participants were right-handed and had normal or corrected-to-normal vision and normal color vision, and none of them had a history of neurological or psychiatric illness. 


\section{Materials}

\section{Facial Dissatisfaction Measure}

The Negative Physical Self Scale (NPSS) has 48 items and is comprised of five subscales, including general appearance, face, fatness, shortness and thinness concern (Chen et al., 2006b; Chen and Jackson, 2007). The 11-item facial concern subscale (NPSSFace) assessing thoughts, feelings, and behaviors related to face concerns was used to identify HFD and LFD women. The items were rated from 0 ("not at all like me") to 4 ("very much like me"). The NPSS-Face was reliable $(\alpha=0.85)$, stable over 9 months among girls $(r=0.62)$ from middle school to high school and had satisfactory convergent and predictive validity among samples of adolescents and young adults (Chen et al., 2006b; Chen and Jackson, 2007). In this research, its Cronbach coefficient was $\alpha=0.964$.

\section{Stimuli}

The photographic stimuli consisted of 18 attractive female face pictures, 18 unattractive female face pictures, and 36 neutral vase pictures. These pictures were adapted from a previous study (Zhang et al., 2010). They were cropped and sized into a uniform size $\left(228^{*} 228\right.$ pixel), which were displayed in black and white. Grayscale images were used. Furthermore, their hair, ear and neck were excluded by Photoshop. These standardized face pictures were assessed in arousal, pleasure and attractiveness by 45 undergraduate females who did not participate in the eye movement (EM) experiment and were required to respond on a five-point Likert scale from 1-not a bit to 5-very. Consequently, 18 attractive face pictures and 18 unattractive face pictures were selected. A paired-sample $t$-test on the two types of pictures revealed that there was no significant difference in arousal, $t=-1.201$, $d f=17, p=0.246$ (unattractive faces $M=3.68$, attractive faces $M=3.77 ; M D=-0.09, S D=0.33$ ), but there was a significant difference in pleasure, $t=-4.20, d f=17$, $p=0.001$ (unattractive faces $M=2.78$, attractive faces $M=3.22$; $M D=-0.44, S D=0.10)$, and attractiveness, $t=-6.50, d f=17$, $p<0.001$ (unattractive faces $M=2.72$; attractive faces $M=3.33$; $M D=-0.62, S D=0.40)$. Finally, 18 attractive-neutral picture pairs $(A-N), 18$ unattractive-neutral picture pairs $(\mathrm{U}-\mathrm{N}), 18$ attractive-unattractive picture pairs $(\mathrm{A}-\mathrm{U})$ and 18 neutral-neural picture pairs $(\mathrm{N}-\mathrm{N})$ were yielded. Examples of the stimuli are listed in Figure 1.

\section{Apparatus}

Eye movements were registered during the viewing period by an Eye Link II tracker (SR Research, Mississauga, ON, Canada) connected to a host computer. The sampling rate of the pupiltracking mode was $1000 \mathrm{~Hz}$, and the spatial resolution was $0.1^{\circ}$. The participants were seated approximately $60 \mathrm{~cm}$ in front of a 19 -inch, $85-\mathrm{Hz}$ monitor connected to a Pentium IV $3.2-\mathrm{GHz}$ host computer and were provided with a chin rest to keep the viewing distance constant and minimize head movements. The participants were required to complete a standardized calibration procedure for EMs prior to the experiment, in which they were required to fixate on nine white dots randomly appearing on the black screen background in a $3 \times 3$ array.

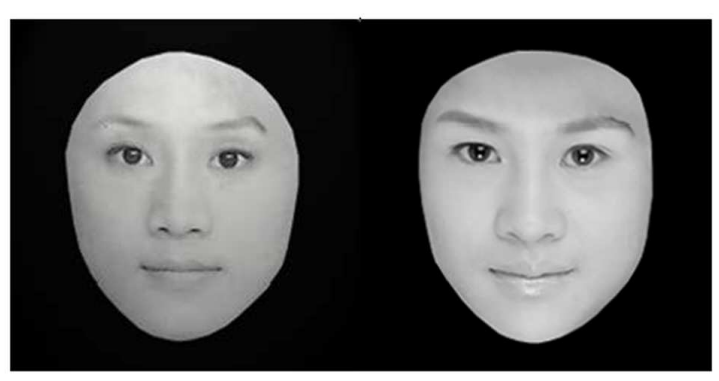

(1)

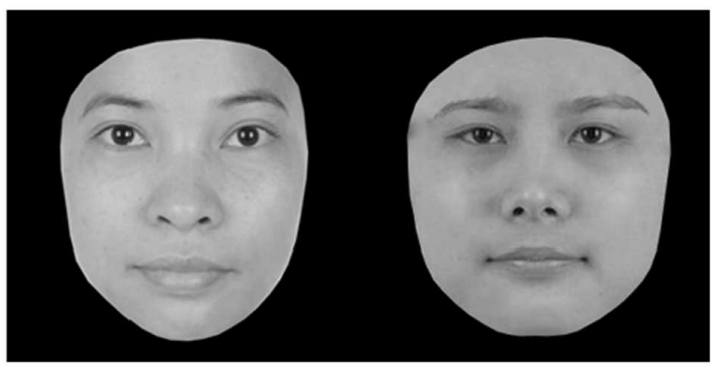

(2)

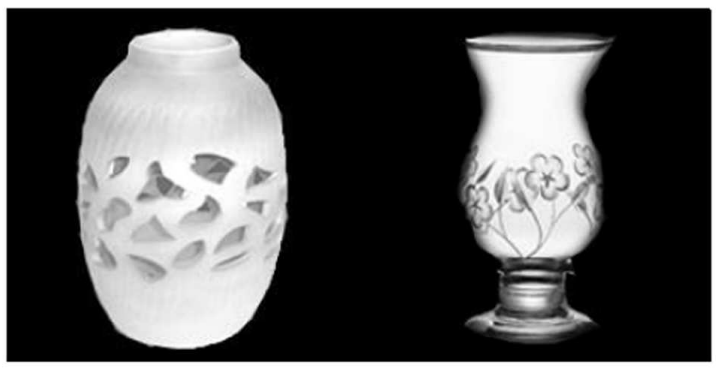

(3)

FIGURE 1 | Attractive faces (1), unattractive faces (2), and vase sample (3).

\section{Procedure}

The study was approved by the ethics committee for human research at Southwest University. Upon arrival at our laboratory, participants signed an informed consent form to learn about the aim of this study, which was to examine how EMs would be affected when viewing pictures. The task would last for about $30 \mathrm{~min}$ and participants were paid 10 Yuan as compensation for their time after the task. Subsequently, the participants were seated in front of the host computer and were asked to lay their chin on a chin-rest. They could regulate the height of the chin-rest accordingly to achieve a comfortable state.

Upon completion of standard calibration for their eye gaze, participants were required to complete the dot-probe task. At the beginning of each trial, a fixation cross was presented in the center of the screen for $1000 \mathrm{~ms}$ to prepare participants for the following procedures. Then, random picture pairs were immediately displayed for $1500 \mathrm{~ms}$, followed by replacement of one of the pictures by a black dot $(\bullet)$. Each picture pair was 
presented twice so that each picture location was counterbalanced by presenting them on both sides of the computer screen twice. When the fixation cross was presented, participants was asked to have to fixate the cross, viewed subsequent pictures and pressed one key (A) when the dots were located on the left side of the screen and another key (L) when the dots were on the right side as quickly as possible. Each probe appeared until a response was made within $5 \mathrm{~s}$, followed by a blank screen for $300 \mathrm{~ms}$. In total, 144 trials were performed in this experiment.

\section{Analysis Eye Movement Data}

Saccades that remained stable within a $1^{\circ}$ visual angle for $100 \mathrm{~ms}$ or longer were defined as fixations to that position, the duration of which was recorded (Gao et al., 2011b). Fixations on either picture in each pair were effective when the following two conditions were satisfied: (i) Participants fixated in the central region (fixation cross) before picture onset, (ii) Saccades occurred at least $100 \mathrm{~ms}$ after picture onset and before picture offset (Fischer and Weber, 1993; Gao et al., 2011b). In this study, initial fixations were made on one of the pictures in $92.97 \%$ of the trials. The EM data were analyzed using the Eyenal Data Analysis Program (Applied Science Group 2000). There are four EM indices, including direction bias, first fixation latency bias, first fixation duration bias, and overall gaze duration bias. They can be calculated using the methods displayed in Table 1 (Garner et al., 2006; Castellanos et al., 2009). Then, we used 2 (Group: HFD vs. LFD women $) \times 2$ (Picture Type: attractive vs. unattractive faces) repeated measures ANOVA analysis with the abovementioned four EM indices as dependent variables.

\section{RT Data}

According to a previous study (Koster et al., 2004), the attention disengagement index $=[(\mathrm{BlDr}+\mathrm{BrDl}) / 2-(\mathrm{NDl}-\mathrm{NDr}) / 2] / 2(\mathrm{~B}$ : face pictures in the A-N and $\mathrm{U}-\mathrm{N}$ pairs condition; $\mathrm{D}$ : the location of the probe; $\mathrm{N}$ : neutral pictures in the N-N condition; l: left; $\mathrm{r}$ : right). Positive values were indicative of orienting (i.e., faster RT to probes following face pictures than probes following neutral pictures), zero scores denoted no attentional bias, and negative scores reflected avoidance (i.e., slower RT to probes following face pictures than to those following neutral pictures) (Gao et al., 2011b). A 2(Group) × 2(Picture Type) repeated measures ANOVA analysis was also conducted with the scores of attention disengagement index as dependent variables.

\section{RESULTS}

\section{Eye-Movement Data Direction Bias}

Under the condition of $\mathrm{A}-\mathrm{N}$ and $\mathrm{U}-\mathrm{N}$ pairs, a $2 \times 2$ repeated measures ANOVA analysis was conducted with Group as the between-subjects factor (HFD vs. LFD) and Picture Type as the within-subjects factor (attractive vs. unattractive faces). The results demonstrated a significant main effect for Group, $F(1,52)=7.90, p=0.007, \eta=0.13$, and significant interaction, $F(1,52)=4.58, p=0.037, \eta=0.08$ (seen in Table 2 ). A simple effect analysis with Bonferroni adjustment suggested that HFD women directed their first fixations toward unattractive faces more often than attractive faces, $F(1,52)=4.52, p=0.038$, $\eta=0.08$, and also toward unattractive faces more often than LFD women did, $F(1,52)=10.90, p=0.002, \eta=0.17$. Additionally, in the condition of A-U pairs, independent sample $t$-test did not show any significant difference between HFD and LFD groups, $t=-0.15, d f=50, p=0.880$.

\section{First Fixation Latency Bias}

Under the condition of A-N and U-N pairs, we then investigated the first fixation latency bias using a similar $2 \times 2$ repeated measures ANOVA. The results only revealed a significant interaction effect, $F(1,52)=8.53, p=0.005, \eta=0.14$ (seen in Table 2). A further simple effect with Bonferroni adjustment examination indicated that HFD women obviously directed their first fixation toward unattractive faces more quickly than LFD women did, $F(1,52)=5.68, p=0.021, \eta=0.10$, and also directed unattractive faces more quickly than they did the attractive faces, $F(1,52)=5.74, p=0.020, \eta=0.10$. In addition, an independent sample $t$-test in A-U pairs revealed that there was no significant difference between groups, $t=1.32, d f=50, p=0.19$.

\section{First Fixation Duration Bias}

Under the condition of A-N and U-N pairs, a $2 \times 2$ repeated measures ANOVA revealed that the interaction effect was pronounced, $F(1,52)=8.39, p=0.006, \eta=0.13$ (seen in Table 2). Simple effect analysis with Bonferroni adjustment indicated that HFD women were prone to fixate their initial gaze longer on unattractive faces than attractive ones, $F(1,52)=3.79$, $p=0.057, \eta=0.07$, whereas LFD women showed the opposite mode, $F(1,52)=4.62, p=0.036, \eta=0.08$. Moreover, a difference between groups emerged only with unattractive faces, $F(1,52)=5.92, p=0.018, \eta=0.10$. In all, HFD women showed

TABLE 1 | Calculation of eye movement (EM) indices.

\section{EM indices}

Direction bias score

First fixation latency bias score

First fixation duration bias score

Overall gaze duration bias score

\section{Calculation}

The number of trials in which first eye-movements were directed toward attractive or unattractive faces/total trials

The first fixation latency of attractive or unattractive faces - the first fixation latency of neutral pictures

The first fixation duration on attractive or unattractive faces - the first fixation duration on neutral pictures

The total time spent gazing at attractive or unattractive faces/the total time of gazing at face and neutral pictures 
initial attention maintenance to unattractive faces, whereas LDF women merely focused on attractive faces. At last, under the condition of A-U pairs, an independent sample $t$-test revealed that there was no significant difference between HFD and LFD groups, $t=1.43, d f=50, p=0.158$.

\section{Overall Gaze Duration Bias}

Under the condition of A-N and U-N pairs, total gaze duration scores were analyzed using a $2 \times 2$ repeated measures ANOVA to investigate attention maintenance. It was found that only the main effect of Picture Type was significant, $F(1,52)=25.19$, $p<0.001, \eta=0.33$ (seen in Table 2), suggesting that all participants fixated significantly longer on attractive faces than unattractive faces. Ultimately, an independent sample $t$-test did not show any significant difference between groups in A-U pairs, $t=0.25, d f=50, p=0.803$.

\section{RT Data}

The attention disengagement scores were analyzed using a $2 \times 2$ repeated measures ANOVA under the condition of A-N and U-N pairs. The main effect of Group and the interaction effect reached significance, $F(1,52)=25.65, p<0.001, \eta=0.33, F(1,52)=5.17$, $p=0.027, \eta=0.09$ (seen in Figure 2). A simple effect analysis with Bonferroni adjustment indicated that the attention disengagement scores of HFD women, whether on attractive faces or on unattractive ones, were greater than the attention disengagement scores of LFD women, $F(1,52)=4.72, p=0.034$, $\eta=0.08, F(1,52)=38.98, p<0.001, \eta=0.43$; however, only the attention disengagement scores of attractive faces among LFD women were greater than the attention disengagement scores of unattractive faces, $F(1,52)=8.68, p=0.005, \eta=0.14$,

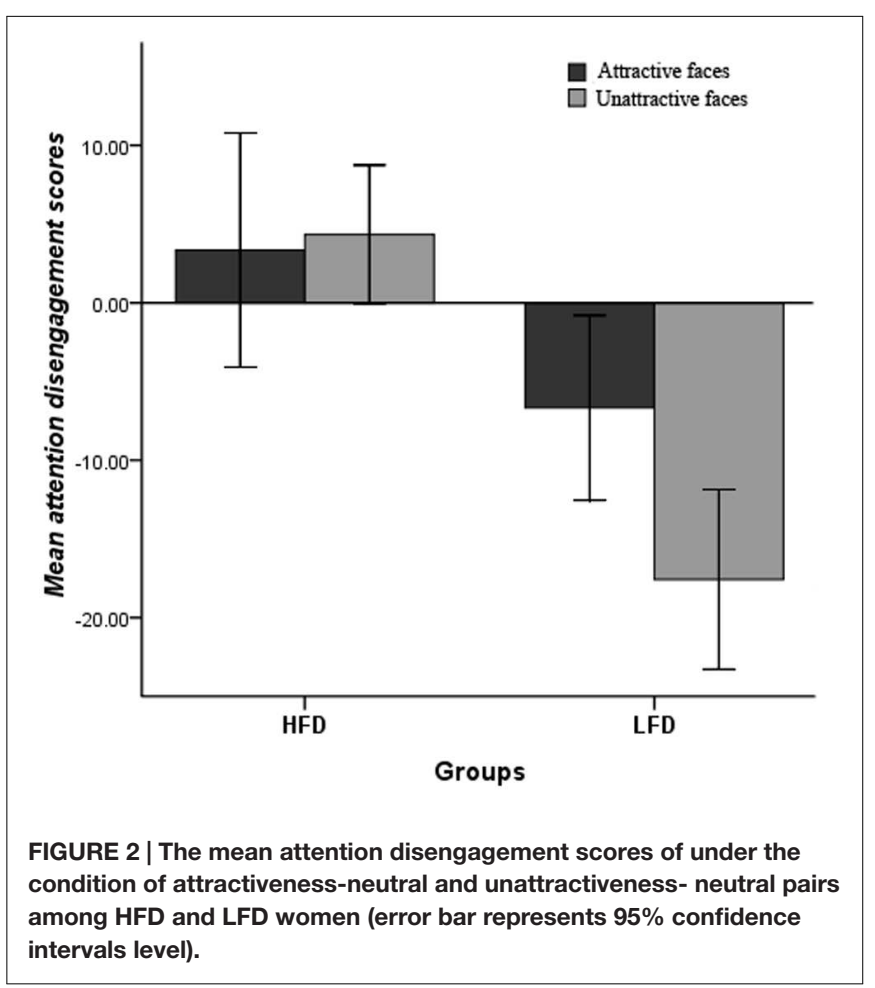


whereas there was no significant difference among HFD women, $F(1,52)=0.07, p=0.790, \eta=0.001$. Compared to LFD women, HFD women showed attention maintenance to both unattractive and attractive faces.

In addition, the attentional disengagement index of unattractive faces in A-U pairs was analyzed using an independent sample $t$-test, demonstrating no significant difference in groups, $t=0.74, d f=52, p=0.315$ (Table 3).

\section{DISCUSSION}

The purpose of the study was to assess spatial attention biases among HFD and LFD women by directly tracking their EM. To the best of our knowledge, this is the first experimental study to examine attention orienting-avoidance and disengagementmaintenance to attractive/unattractive faces among HFD and LFD women. Our hypothesis was partly supported by our results. Compared to LFD women, HFD women were more likely to locate their initial fixation on unattractive faces, directed their first gaze faster to them, and had longer first fixation on them, consistent with our hypothesis, but HFD women did not have shorter first fixation on attractive faces or longer total gaze duration on unattractive faces.

\section{Attention Orienting: Direction Bias and First Fixation Latency Bias}

Regarding the A-N and U-N pairs, HFD women directed their first fixations toward both types of faces more often than the LFD group did and had more initial fixations on unattractive faces than attractive faces, which proved our hypothesis. Furthermore, HFD women directed their first fixation toward unattractive faces faster than LFD women did and directed their first fixation towards unattractive faces more quickly than they did the attractive faces, which also supported our hypothesis. These results suggested attention orienting of unattractive faces among HFD women. Consistent with this study, some previous researchers found that weight dissatisfied female showed similar biases in initial orientation characterized by more frequent and faster first fixations on fatness-related information (e.g., fat words and fat pictures) than on neutral stimuli (Gao et al., 2011b, 2012). Specifically, in an ERP study, Gao et al. (2011a) claimed that the early anterior N100 and bilateral parietal and occipital N170 amplitudes elicited by fatness-related words were larger than those elicited by thinness-related and neutral words among weight dissatisfied females. In addition, the amplitudes of N170 and N100 were larger for the negative words because the females were influenced by the valence of emotion (Doallo et al., 2007). According to the cognitive-behavioral model concerning body image dissatisfaction proposed by Vitousek and Hollon's (1990), attention bias toward body-related stimuli arose from underlying maladaptive self-schemata associated with shape and weight. Furthermore, Perpiñá et al. (1993) found that individuals with maladaptive body schemata were different from others in several ways, including facilitation of attention and memory processing for schema-consistent or schema-related stimulus (e.g., fatnessrelated or body-related stimulus) (Perpiñá et al., 1993). Based on this model, we guessed that spatial attentional bias toward unattractive faces might derive from underlying maladaptive self-schemata associated with facial attractiveness, which could facilitate the attentional processing of unattractive faces among HFD women.

Concerning A-U pairs, all participants did not show any direction bias and first fixation latency bias toward unattractive faces. One reason might be that faces are generally attentiongrabbing (Ro et al., 2001; Mack et al., 2002; Theeuwes and Van der Stigchel, 2006; Bindemann et al., 2007). When faces are presented along with vases, the face might initially capture attention, while the attractiveness or unattractiveness of the face can further modulate the overt attentional selection of the face. When two faces are presented, this might be a more complex process, because the available attentional resources are more strongly distracted by the other, concurrent face. Consequently, when an attractive face and an unattractive face are displayed simultaneously on a computer screen, participants did not show attention orienting or avoidance to any faces in the condition of A-U pairs.

\section{Attention Maintenance: First Fixation Duration Bias and Overall Gaze Duration Bias}

Under the condition of A-N and U-N pairs, HFD women showed initial attention maintenance to unattractive faces, whereas LDF women merely focused on attractive faces. Our hypothesis was partly supported because attention avoidance to attractive faces among HFD women did not emerge in our study. However, the result was in line with a previous study (Gao et al., 2012), reporting that attention avoidance of thin body pictures was not found among weight dissatisfied females. In addition, our result here was consistent with a previous study investigating BDD individuals. They implied that during a long stimulus presentation $(1000 \mathrm{~ms})$, individuals with dysmorphic concern attended to both faces and face-related images, whereas during a short stimulus presentation $(200 \mathrm{~ms})$, they focused on disgusting images (Onden-Lim et al., 2012). Furthermore, similar to a previous study with weight dissatisfied female (Gao et al., 2011b), HFD women were indeed prone to focus longer on unattractive faces than attractive faces, which may indicate that once attention was captured by unattractive faces in the early stages of attentional processing, HFD women might have difficulty, at least initially, in disengaging from unattractive faces. Fox et al. (2002) considered that attentional bias may consist of two phases. In the first orientation phase, individuals are sensitive to threatening stimulus and attention is drawn to the threat stimulus. Second, threatening stimulus could influence the maintenance of attention or the participants' ability to carry out attention disengagement (Fox et al., 2002). Accordingly, HFD women may regard unattractive faces as threatening stimulus and have difficulty in disengaging attention from unattractive faces. Evidence from behavioral data, in which HFD women had difficulty in disengaging from unattractive faces in the condition of $A-N$ and $U-N$ pairs, also proved this observed phenomenon. However, in 
TABLE 3 | Reaction time (in ms) for probe among high face dissatisfaction (HFD) and low face dissatisfaction (LFD) women.

\begin{tabular}{|c|c|c|c|c|c|}
\hline \multirow[b]{2}{*}{ Face pictures position } & \multirow[b]{2}{*}{ Probe position } & \multicolumn{2}{|c|}{ HFD } & \multicolumn{2}{|c|}{ LFD } \\
\hline & & $M$ & $S D$ & $M$ & $S D$ \\
\hline \multicolumn{6}{|l|}{ Attractiveness-neutral } \\
\hline Left & Left & 511 & 105 & 512 & 82 \\
\hline Left & Right & 520 & 122 & 495 & 85 \\
\hline Right & Left & 524 & 125 & 521 & 105 \\
\hline Right & Right & 484 & 109 & 494 & 80 \\
\hline \multicolumn{6}{|c|}{ Unattractiveness-neutral } \\
\hline Left & Left & 520 & 119 & 529 & 99 \\
\hline Left & Right & 501 & 497 & 497 & 70 \\
\hline Right & Left & 520 & 132 & 506 & 103 \\
\hline Right & Right & 494 & 108 & 487 & 74 \\
\hline
\end{tabular}

the condition of A-U pairs, both EM data and behavioral data showed there was no difference between HFD and LFD women.

Analyses for overall fixation duration bias indicated that both groups had overall attention maintenance to attractive and unattractive faces relative to neutral stimulus. Moreover, both groups had more difficulty disengaging their overall attention from attractive faces than unattractive faces. Although the result was incongruent with previous research (Gao et al., 2012) indicating that weight dissatisfied women had overall gaze duration bias toward fat body-related pictures, some researchers found that faces had an advantage in retaining attention over other stimulus categories (Bindemann et al., 2005). Human beings are born to prefer face stimulus (Johnson et al., 1991; Valenza et al., 1996; Slater and Quinn, 2001), because faces convey social and biological implications, such as identity (age, gender etc.) and sex. Therefore, all participants fixated their attention on faces, whether they were attractive or not. Moreover, researchers found that people showed preference for attractive faces as early as during infancy (Langlois et al., 1990; Rubenstein et al., 1999). Moreover, behavioral studies have revealed that both HFD and LFD women focused longer on attractive faces than unattractive faces (Aharon et al., 2001). Brain imaging studies have revealed that attractive faces possess rewarding value and activated reward circuit and emotion-related brain regions, such as the OFC, amygdala and basal ganglia (O’Doherty et al., 2003). This could explain why HFD women showed overall attention maintenance, instead of avoidance, to attractive faces.

\section{Limitation and Prospection}

Despite some interesting findings, this research contains several limitations. Firstly, although the study focused on undergraduate females with facial dissatisfaction, the findings may not generalize to undergraduate male and adolescent samples. Therefore, in future research, replications among samples from other age and gender groups are needed to assess the consistency and difference of such an attention model across gender and age. Secondly, the findings may not apply completely to non-Chinese samples. Chen and Jackson (2006), using a lexical decision task with subliminal priming, found that American participants with weight dissatisfaction judged self-primed positive body words more slowly than self-primed negative body words, while participants with relatively positive body image showed a contrary tendency. However, the matched Chinese participants did not show such a trend (Chen and Jackson, 2006). It is possible that there exists cultural diversity in attentional bias toward face pictures among HFD groups. Hence, extensions to samples of other cultural backgrounds are also one of our future research directions. Lastly, face pictures were displayed in black/white but not in color. Given that colored pictures are ecologically more valid, future studies employing colored pictures may promote the ecological validity of our findings.

\section{CONCLUSION}

In summary, HFD women first showed attention orienting toward unattractive faces but did not show attention avoidance to attractive pictures. Additionally, HFD women, similar to LFD women, displayed difficulty in overall attention disengagement from attractive faces, but there weren't any attention bias found in attractive - unattractive face pairs.

\section{AUTHOR CONTRIBUTIONS}

HK and YS conceived of the study, participated in its design and coordination, performed the measurement and drafted the manuscript together; HC participated in the design and interpretation of the data; $\mathrm{TB}$ participated in revising the manuscript critically for important intellectual content; XG participated in the statistical analysis. All authors read and approved the final manuscript.

\section{ACKNOWLEDGMENTS}

This research was supported by grants to the corresponding authors from the National Natural Science Foundation of China (31170981), Education Ministry of China, and to a co-author from Technology Innovation Fund for Southwest University graduate students (kb2011001). 


\section{REFERENCES}

Aharon, I., Etcoff, N., Ariely, D., Chabris, C. F., O’Connor, E., and Breiter, H. C. (2001). Beautiful faces have variable reward value: fMRI and behavioral evidence. Neuron 32, 537-551. doi: 10.1016/S0896-6273(01)00491-3

Ben-Tovim, D. I., and Walker, M. K. (1991). Further evidence for the Stroop test as a quantitative measure of psychopathology in eating disorders. Int. J. Eat. Disord. 10, 609-613. doi: 10.1002/1098-108X(199109)10:5<609::AIDEAT2260100513>3.0.CO;2-M

Bindemann, M., Burton, A. M., Hooge, I. T., Jenkins, R., and de Haan, E. H. (2005). Faces retain attention. Psychon. Bull. Rev. 12, 1048-1053. doi: 10.3758/BF03206442

Bindemann, M., Burton, A. M., Langton, S. R., Schweinberger, S. R., and Doherty, M. J. (2007). The control of attention to faces. J. Vis. 7, 1-8. doi: 10.1167/ 7.10.15

Buhlmann, U., McNally, R. J., Wilhelm, S., and Florin, I. (2002). Selective processing of emotional information in body dysmorphic disorder. J. Anxiety Disord. 16, 289-298. doi: 10.1016/S0887-6185(02)00100-7

Caseras, X., Garner, M., Bradley, B. P., and Mogg, K. (2007). Biases in visual orienting to negative and positive scenes in dysphoria: an eye movement study. J. Abnorm. Psychol. 116, 491-497. doi: 10.1037/0021-843X. 116.3.491

Castellanos, E. H., Charboneau, E., Dietrich, M. S., Park, S., Bradley, B. P., Mogg, K., et al. (2009). Obese adults have visual attention bias for food cue images: evidence for altered reward system function. Int. J. Obes. 33, 1063-1073. doi: 10.1038/ijo.2009.138

Chen, H., Feng, W., and Huang, X. (2006a). Body beauty: characteristics of ideal physical self among adolescent in China. J. Southwest China Norm. Univ. 32, $12-17$

Chen, H., and Jackson, T. (2006). Differential processing of self-referenced versus other-referenced body information among American and Chinese young adults with body image concerns. Eat. Behav. 7, 152-160. doi: 10.1016/j.eatbeh.2005.08.012

Chen, H., and Jackson, T. (2007). Stability of body image concerns among Chinese adolescents: nine-month test-retest reliabilities of the Negative Physical Self Scale. Percept. Mot. Skills 105, 677-680. doi: 10.2466/pms.105.2.677-680

Chen, H., Jackson, T., and Huang, X. (2006b). The Negative Physical Self Scale: initial development and validation in samples of Chinese adolescents and young adults. Body Image 3, 401-412. doi: 10.1016/j.bodyim.2006.07.005

Cororve, M. B., and Gleaves, D. H. (2001). Body dysmorphic disorder: a review ofconceptualizations, assessment, and treatment strategies. Clin. Psychol. Rev. 21, 949-970. doi: 10.1016/S0272-7358(00)00075-1

Desrumaux, P., De Bosscher, S., and Leoni, V. (2009). Effects of facial attractiveness, gender, and competence of applicants on job recruitment. Swiss J. Psychol. 68, 33-42. doi: 10.1024/1421-0185.68.1.33

Didie, E. R., Kuniega-Pietrzak, T., and Phillips, K. A. (2010). Body image in patients with body dysmorphic disorder: evaluations of and investment in appearance, health/illness, and fitness. Body Image 7, 66-69. doi: 10.1016/j.bodyim.2009.09.007

Doallo, S., Cadaveira, F., and Holguín, S. R. (2007). Time course of attentional modulations on automatic emotional processing. Neurosci. Lett. 418, 111-116. doi: 10.1016/j.neulet.2007.03.009

Feng, W., Luo, W., Liao, Y., Chen, H., and Luo, Y. (2010). Attention biases of undergraduate women with fat negative physical self: orienting or maintenance. Acta Psychol. Sin. 42, 779-790. doi: 10.3724/SP.J.1041.2010.00779

Fischer, B., and Weber, H. (1993). Express saccades and visual attention. Behav. Brain Sci. 16, 553-567. doi: 10.1017/S0140525X00031575

Fox, E., Russo, R., and Dutton, K. (2002). Attentional bias for threat: evidence for delayed disengagement from emotional faces. Cogn. Emot. 16, 355-379. doi: 10.1080/02699930143000527

Gao, X., Deng, X., Chen, N., Luo, W., Hu, L., Jackson, T., et al. (2011a). Attentional biases among body-dissatisfied young women: an ERP study with rapid serial visual presentation. Int. J. Psychophysiol. 82, 133-142. doi: 10.1016/j.ijpsycho.2011.07.015

Gao, X., Wang, Q., Chen, H., Wang, B., and Zhao, G. (2012). Time course of attentional bias components toward body-shape related pictures among women with fat negative physical self: an eye movement study. Acta Psychol. Sin. 44, 498-510. doi: 10.3724/SP.J.1041.2012.00498
Gao, X., Wang, Q., Jackson, T., Zhao, G., Liang, Y., and Chen, H. (2011b). Biases in orienting and maintenance of attention among weight dissatisfied women: an eye-movement study. Behav. Res. Ther. 49, 252-259. doi: 10.1016/j.brat.2011.01.009

Garner, M., Mogg, K., and Bradley, B. P. (2006). Orienting and maintenance of gaze to facial expressions in social anxiety. J. Abnorm. Psychol. 115, 760-770. doi: 10.1037/0021-843X.115.4.760

Grocholewski, A., Kliem, S., and Heinrichs, N. (2012). Selective attention to imagined facial ugliness is specific to body dysmorphic disorder. Body Image 9, 261-269. doi: 10.1016/j.bodyim.2012.01.002

Jansen, A., Nederkoorn, C., and Mulkens, S. (2005). Selective visual attention for ugly and beautiful body parts in eating disorders. Behav. Res. Ther. 43, 183-196. doi: 10.1016/j.brat.2004.01.003

Johnson, M. H., Dziurawiec, S., Ellis, H., and Morton, J. (1991). Newborns' preferential tracking of face-like stimuli and its subsequent decline. Cognition 40, 1-19. doi: 10.1016/0010-0277(91)90045-6

Koster, E. H., Crombez, G., Verschuere, B., and De Houwer, J. (2004). Selective attention to threat in the dot probe paradigm: differentiating vigilance and difficulty to disengage. Behav. Res. Ther. 42, 1183-1192. doi: 10.1016/j.brat.2003.08.001

Langlois, J. H., Roggman, L. A., and Rieser-Danner, L. A. (1990). Infants' differential social responses to attractive and unattractive faces. Dev. Psychol. 26, 153-159. doi: 10.1037/0012-1649.26.1.153

Lee, M., and Shafran, R. (2004). Information processing biases in eating disorders. Clin. Psychol. Rev. 24, 215-238. doi: 10.1016/j.cpr.2003.10.004

Liang, Y., Chen, H., Qiu, J., Gao, X., and Zhao, T. (2008). Memory bias toward body information in women with negative physical self: evidence from an ERP study. Acta Psychol. Sin. 40, 913-919. doi: 10.3724/SP.J.1041.2008.00913

Mack, A., Pappas, Z., Silverman, M., and Gay, R. (2002). What we see: inattention and the capture of attention by meaning. Conscious. Cogn. 11, 488-506. doi: $10.1016 /$ S1053-8100(02)00028-4

McNulty, J. K., Neff, L. A., and Karney, B. R. (2008). Beyond initial attraction: physical attractiveness in newlywed marriage. J. Fam. Psychol. 22, 135-143. doi: 10.1037/0893-3200.22.1.135

O'Doherty, J., Winston, J., Critchley, H., Perrett, D., Burt, D. M., and Dolan, R. J. (2003). Beauty in a smile: the role of medial orbitofrontal cortex in facial attractiveness. Neuropsychologia 41, 147-155. doi: 10.1016/S00283932(02)00145-8

Onden-Lim, M., Wu, R., and Grisham, J. R. (2012). Body image concern and selective attention to disgusting and non-self appearance-related stimuli. Body Image 9, 535-538. doi: 10.1016/j.bodyim.2012.07.005

Perpiñá, C., Hemsley, D., Treasure, J., and De Silva, P. (1993). Is the selective information processing of food and body words specific to patients with eating disorders. Int. J. Eat. Disord. 14, 359-366. doi: 10.1002/1098108X(199311)14:3<359::AID-EAT2260140314>3.0.CO;2-G

Posner, M. I., and Petersen, S. E. (1990). The attention system of the human brain. Annu. Rev. Neurosci. 13, 25-42. doi: 10.1146/annurev.ne.13.030190. 000325

Ro, T., Russell, C., and Lavie, N. (2001). Changing faces: a detection advantage in the flicker paradigm. Psychol. Sci. 12, 94-99. doi: 10.1111/1467-9280.00317

Rubenstein, A. J., Kalakanis, L., and Langlois, J. H. (1999). Infant preferences for attractive faces: a cognitive explanation. Dev. Psychol. 35, 848-855. doi: 10.1037/0012-1649.35.3.848

Rumsey, N., and Harcourt, D. (2005). The Psychology of Appearance. Maidenhead: McGraw-Hill International.

Slater, A., and Quinn, P. C. (2001). Face recognition in the newborn infant. Infant Child Dev. 10, 21-24. doi: 10.1002/icd.241

Theeuwes, J., and Van der Stigchel, S. (2006). Faces capture attention: evidence from inhibition of return. Vis. Cogn. 13, 657-665. doi: $10.1080 / 13506280500410949$

Thornhill, R., and Gangestad, S. W. (1999). Facial attractiveness. Trends Cogn. Sci. 3, 452-460. doi: 10.1016/S1364-6613(99)01403-5

Valenza, E., Simion, F., Cassia, V. M., and Umiltà, C. (1996). Face preference at birth. J. Exp. Psychol. Hum. Percept. Perform. 22, 892-903. doi: 10.1037/00961523.22.4.892

Vitousek, K. B., and Hollon, S. D. (1990). The investigation of schematic content and processing in eating disorders. Cogn. Ther. Res. 14, 191-214. doi: 10.1007/BF01176209 
Zhang, Y., Kong, F., Chen, H., Xiang, Y., Gao, X., and Chen, M. (2010). Cognitive bias toward female facial attractiveness in males: evidences from an ERP study. Acta Psychol. Sin. 42, 1060-1072. doi: 10.3724/SP.J.1041.2010.01060

Conflict of Interest Statement: The authors declare that the research was conducted in the absence of any commercial or financial relationships that could be construed as a potential conflict of interest.
Copyright (C) $2016 \mathrm{Kou}, \mathrm{Su}, \mathrm{Bi}, \mathrm{Gao}$ and Chen. This is an open-access article distributed under the terms of the Creative Commons Attribution License (CC BY). The use, distribution or reproduction in other forums is permitted, provided the original author(s) or licensor are credited and that the original publication in this journal is cited, in accordance with accepted academic practice. No use, distribution or reproduction is permitted which does not comply with these terms. 